\title{
Perfect one-dimensional chiral states in biased twisted bilayer graphene
}

DOI:

10.1103/PhysRevB.101.125409

Document Version

Final published version

Link to publication record in Manchester Research Explorer

\section{Citation for published version (APA):}

Tsim, B., Nguyen, N. T., \& Koshino, M. (2020). Perfect one-dimensional chiral states in biased twisted bilayer graphene. Physical Review B, 101(125409). https://doi.org/10.1103/PhysRevB.101.125409

\section{Published in:}

Physical Review B

\section{Citing this paper}

Please note that where the full-text provided on Manchester Research Explorer is the Author Accepted Manuscript or Proof version this may differ from the final Published version. If citing, it is advised that you check and use the publisher's definitive version.

\section{General rights}

Copyright and moral rights for the publications made accessible in the Research Explorer are retained by the authors and/or other copyright owners and it is a condition of accessing publications that users recognise and abide by the legal requirements associated with these rights.

\section{Takedown policy}

If you believe that this document breaches copyright please refer to the University of Manchester's Takedown Procedures [http://man.ac.uk/04Y6Bo] or contact uml.scholarlycommunications@manchester.ac.uk providing relevant details, so we can investigate your claim.

\section{OPEN ACCESS}




\title{
Perfect one-dimensional chiral states in biased twisted bilayer graphene
}

\author{
Bonnie Tsim $\odot,{ }^{1,2}$ Nguyen N. T. Nam, ${ }^{3}$ and Mikito Koshino $\oplus^{4}$ \\ ${ }^{1}$ National Graphene Institute, University of Manchester, Manchester M13 9PL, United Kingdom \\ ${ }^{2}$ Department of Physics and Astronomy, University of Manchester, Manchester M13 9PL, United Kingdom \\ ${ }^{3}$ Mathematics for Advanced Materials Open Innovation Lab, AIST, Sendai 980-8577, Japan \\ ${ }^{4}$ Department of Physics, Osaka University, Toyonaka 560-0043, Japan
}

(Received 18 January 2020; accepted 25 February 2020; published 9 March 2020)

\begin{abstract}
We theoretically study the electronic structure of small-angle twisted bilayer graphene with a large potential asymmetry between the top and bottom layers. We show that the emergent topological channels known to appear on the triangular AB-BA domain boundary do not actually form a percolating network, but instead, they provide independent, perfect one-dimensional eigenmodes propagating in three different directions. Using the continuum-model Hamiltonian, we demonstrate that an applied bias causes two well-defined energy windows which contain sparsely distributed one-dimensional eigenmodes. The origin of these energy windows can be understood using a two-band model of the intersecting electron and hole bands of single-layer graphene. We also use the tight-binding model to implement the lattice deformations in twisted bilayer graphene and discuss the effect of lattice relaxation on the one-dimensional eigenmodes.
\end{abstract}

DOI: 10.1103/PhysRevB.101.125409

\section{INTRODUCTION}

Twisted bilayer graphene (TBG) consists of two layers of graphene overlaid on top of each other with a relative twist between the directions of their crystallographic axes. The moiré interference pattern which emerges from the overlap of the two mismatched graphene lattices results in a strong modification of the electronic structure by the superlattice band folding [1-10]. The system has been shown to exhibit many interesting physical phenomena, and following the realization of superconductivity and correlated insulating states in magic-angle TBG [11-13], there has been a huge surge of theoretical and experimental research in this field.

In this paper, we theoretically study the electronic structure of small-angle TBG with a large interlayer bias (i.e., potential asymmetry between the top and bottom layers) and demonstrate the formation of perfect one-dimensional (1D) states within well-defined energy windows on either side of zero energy. The effect of the interlayer bias on TBG was investigated in previous theoretical works [14-21], and it was found that a large enough bias gives rise to a network of topological channels on the domain boundaries between $\mathrm{AB}$ and BA stacking regions [15,17-21]. There the electronic states at $\mathrm{AB}$ and $\mathrm{BA}$ regions are locally gapped out by the interlayer bias [22], and two topological modes per single spinvalley sector necessarily appear on each AB-BA boundary, as shown in previous works [23-29]. The $\mathrm{AB}$ and $\mathrm{BA}$ regions have different single-valley Hall conductivities, $+e^{2} / h$ and $-e^{2} / h$, respectively [30], and the difference of $2 e^{2} / h$ results in two boundary modes per spin and per valley. Note that the propagating direction of the two modes is opposite between $K$ and $K^{\prime}$. In TBG, the $\mathrm{AB}$ and $\mathrm{BA}$ regions appear periodically in a hexagonal pattern [31-33] such that the boundary channels form a triangular grid, as illustrated in Fig. 1(a). Recently, the network of the topological channels in TBG was experimentally probed by transport measurements [34-36] and also by scanning tunneling spectroscopy [37].

One may expect that the electronic transport in the topological channels of TBG could be described by a percolation model [38] on a triangular network. In a network model, the incoming modes generally split into two or more outgoing modes in different directions. However, here we show that the topological modes in the biased TBG do not form a twodimensional network, but they are actually independent 1D eigenmodes composed of a serial connection of topological channel sections, as shown in Fig. 1(b). In other words, this is a special case of the network model where the scattering matrix at each vertex exclusively connects the specific incoming and outgoing modes as Fig. 1(b). The modes along different directions are never hybridized, and therefore all these states serve as independent perfect 1D channels over the whole TBG. The result is consistent with a recent work which

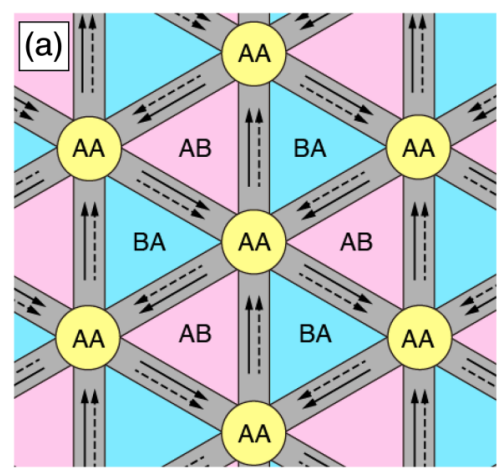

(b)

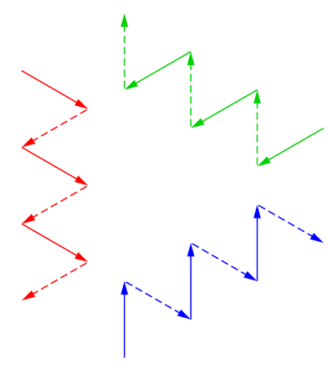

FIG. 1. (a) AB and BA domain structure and topological boundary channels in the biased TBG. Here solid and dashed arrows represent independent traveling modes for the $K$ valley (modes 1 and 2). (b) Independent 1D eigenmodes in three directions. 
predicts the perfect nesting of the Fermi surface in the biased TBG [19].

In the following, we calculate the electronic band structure of the TBG using the continuum-model Hamiltonian and present the band structures for various twist angles and electric field dependencies. The energy band structures show that an applied bias causes two well-defined energy windows which contain sparsely distributed perfect $1 \mathrm{D}$ eigenmodes, separated by a cluster of nearly flat bands around the charge neutrality point. We also use the tight-binding model to implement arbitrary lattice deformations in TBG and discuss the effect of lattice relaxation on the 1D eigenmodes. Last, we explain the origin of these energy windows by a perturbational approach from the small interlayer coupling limit and also by a two-band model consisting of the intersecting electron and hole bands of single-layer graphene. The tunability of the TBG energy dispersion in a perpendicular electric field means there is the potential to explore the parameter space where these 1D eigenmodes can be found in its experimental realization.

This paper is organized as follows: In Sec. II, we introduce the continuum-model Hamiltonian and describe the formation of perfect 1D eigenmodes for varying angles and biases. In Sec. III, we consider the effect of lattice relaxation on the 1D eigenmodes. Last, we explain the origin of $1 \mathrm{D}$ eigenmodes in Sec. IV and present a brief conclusion in Sec. V.

\section{CONTINUUM MODEL}

We calculate the electronic band structure of the twisted bilayer graphene using the continuum model [1,6-8,39-41]. For a small twist angle, the Hamiltonian is given by

$$
H_{\mathrm{TBG}}=\left(\begin{array}{cc}
H_{1} & U^{\dagger} \\
U & H_{2}
\end{array}\right)
$$

where

$$
H_{1}=\left(\begin{array}{cc}
\frac{\Delta}{2} & -v \pi^{\dagger} \\
-v \pi & \frac{\Delta}{2}
\end{array}\right), \quad H_{2}=\left(\begin{array}{cc}
-\frac{\Delta}{2} & -v \pi^{\dagger} \\
-v \pi & -\frac{\Delta}{2}
\end{array}\right),
$$

and

$$
U=u \sum_{j=0,1,2} e^{i \Delta \mathbf{K}_{j} \cdot \mathbf{r}}\left(\begin{array}{cc}
1 & e^{-i \frac{2 \pi}{3} j} \\
e^{i \frac{2 \pi}{3} j} & 1
\end{array}\right)
$$

The Hamiltonian (1) is equivalent to the continuum-model Hamiltonian derived in $[1,6,39]$ up to a gauge transformation $[42,43]$. The on-diagonal blocks describe graphene layers 1 and 2 , where $\pi=\hbar\left(\xi k_{x}+\mathrm{i} k_{y}\right)$, and the valley index $\xi= \pm 1$ is used to distinguish between the $K$ and $K^{\prime}$ valleys. The parameter $v$ is the band velocity of monolayer graphene, where $\hbar v / a=2.1354 \mathrm{eV}$ (the lattice constant of graphene is given by $a=2.46 \AA$ ) $[39,44]$, and $\Delta$ represents the electrostatic energy shift induced by the perpendicular electric field. The off-diagonal blocks describe the moiré interlayer coupling between the two twisted layers, where the interlayer coupling strength is given by $u=0.103 \mathrm{eV}$. The vectors $\Delta \mathbf{K}_{j}(j=$ $0,1,2)$ account for the shift between the original Brillouin zone corners of the two layers and are given by

$$
\Delta \mathbf{K}_{j}=\frac{4 \pi \theta}{3 a}\left[-\sin \left(\frac{2 \pi j}{3}\right), \cos \left(\frac{2 \pi j}{3}\right)\right],
$$

where $\theta$ is the twist angle between the two layers in radians.
We calculate the energy spectrum for the $K$ and $K^{\prime}$ valleys independently as intervalley coupling is negligible at small twist angles. Zone folding is used to bring the states in each valley with momenta connected by the moiré reciprocal lattice vectors, $\mathbf{G}_{1}=\Delta \mathbf{K}_{1}-\Delta \mathbf{K}_{0}$ and $\mathbf{G}_{2}=\Delta \mathbf{K}_{2}-\Delta \mathbf{K}_{0}$. The basis of $k$ states of layers 1 and 2 can be taken to be

$$
\begin{aligned}
& \mathbf{k}_{m_{1}, m_{2}}^{(1)}=\mathbf{k}+\Delta \mathbf{K}_{0}+m_{1} \mathbf{G}_{1}+m_{2} \mathbf{G}_{2}, \\
& \mathbf{k}_{m_{1}, m_{2}}^{(2)}=\mathbf{k}-\Delta \mathbf{K}_{0}+m_{1} \mathbf{G}_{1}+m_{2} \mathbf{G}_{2},
\end{aligned}
$$

respectively, where $\mathbf{k}$ is the wave number in the first moiré Brillouin zone (mBZ) spanned by $\mathbf{G}_{1}$ and $\mathbf{G}_{2}$ and $m_{1}$ and $m_{2}$ are integers. The size of the basis is chosen such that when the Hamiltonian is numerically diagonalized, the energy bands converge up to a cutoff energy.

Figure 2(a) presents the electric field dependence of the TBG band structure for various twist angles, $\theta=$ $1^{\circ}, 0.5^{\circ}, 0.3^{\circ}$, and $0.2^{\circ}$. The band structures include energy bands from both the $K$ (black) and $K^{\prime}$ (red) valleys and are shown for the path $\kappa \rightarrow \gamma \rightarrow \mu \rightarrow \kappa^{\prime}$ in the mBZ illustrated in Fig. 2(b). The original Dirac point of layer 1 is placed at the corner of the $\mathrm{mBZ}$ at $\kappa^{\prime}$, and the original Dirac point of layer 2 is placed at $\kappa$. In increasing $\Delta$, we see that the energy bands gradually shift toward zero energy, forming a cluster of nearly flat bands around the charge neutrality point. At the same time, two well-defined energy windows, where energy bands are only sparsely distributed, are formed above and below the zero-energy band cluster. The size of the energy windows is not strongly affected by the size of $\Delta$, which can be seen for $\theta=0.2^{\circ}$ with increasing $\Delta$.

Most interesting is the formation of 1D propagating modes inside the energy windows, which connect the zero-energy band cluster to the bulk bands outside of the energy windows. Figure 2(c) shows a three-dimensional plot of the bands from the $K$ valley calculated for $\theta=0.5^{\circ}$ and $\Delta=400 \mathrm{meV}$, and Fig. 2(d) is the Fermi surface of the same system at $E_{F}=50 \mathrm{meV}$, where black and red lines represent $K$ and $K^{\prime}$, respectively. We see that the band dispersion of $K$ is actually composed of three intersecting planes, with band velocities parallel to the $(0,-1),(\sqrt{3} / 2,1 / 2)$, and $(-\sqrt{3} / 2,1 / 2)$ directions. The different planes are not hybridized with each other, giving nearly straight Fermi lines at the fixed energy. Such straight Fermi surfaces were also reported in a recent paper [19]. For the largest bias $\Delta=400 \mathrm{meV}$, we notice some flat levels appear in the upper part of the energy window independently of the dispersive 1D states (e.g., three horizontal lines in the range $50 \mathrm{meV}<|E|<100 \mathrm{meV}$ for $\theta=0.3^{\circ}$ ), which can be interpreted as pseudo-Landau levels of the fictitious gauge field [17].

\section{EFFECT OF LATTICE RELAXATION}

The real TBG is not a simple stack of rigid graphene layers as assumed in the previous section, but it has a spontaneous lattice relaxation and resulting $\mathrm{AB} / \mathrm{BA}$ domain formation [31-33,35,45-53]. Such a structural deformation modifies the electronic band structure [19,20,35,44,50,52-54]. Here we calculate the energy band structures in the presence of the lattice strain using the tight-binding method [50]. The 
(a)
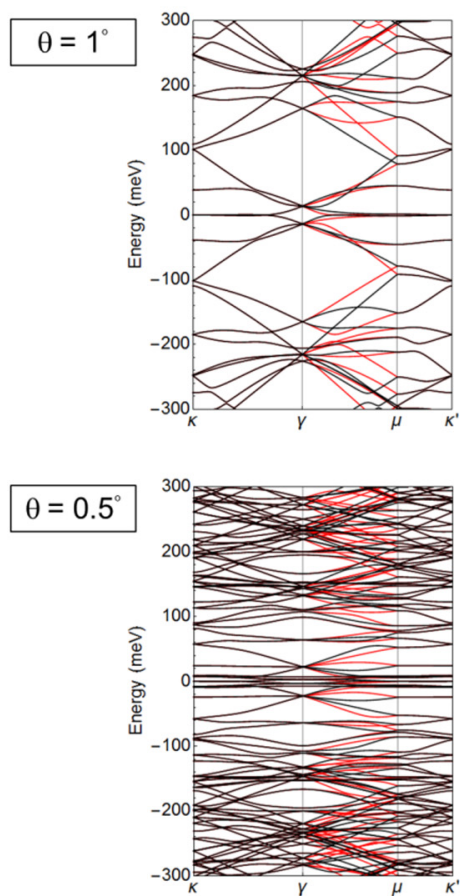

$\theta=0.3^{\circ}$

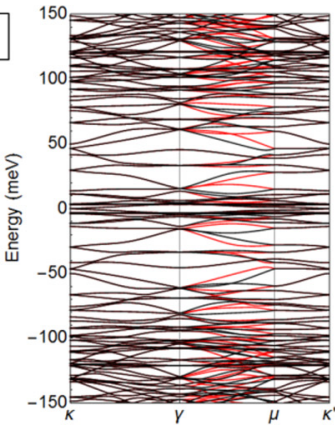

$\theta=0.2^{\circ}$

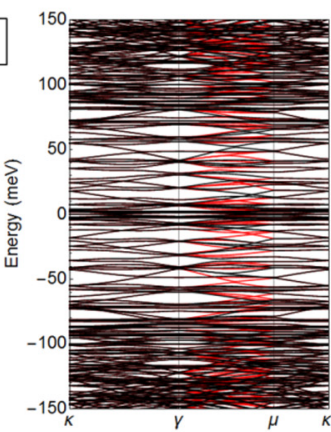

$\Delta=200 \mathrm{meV}$
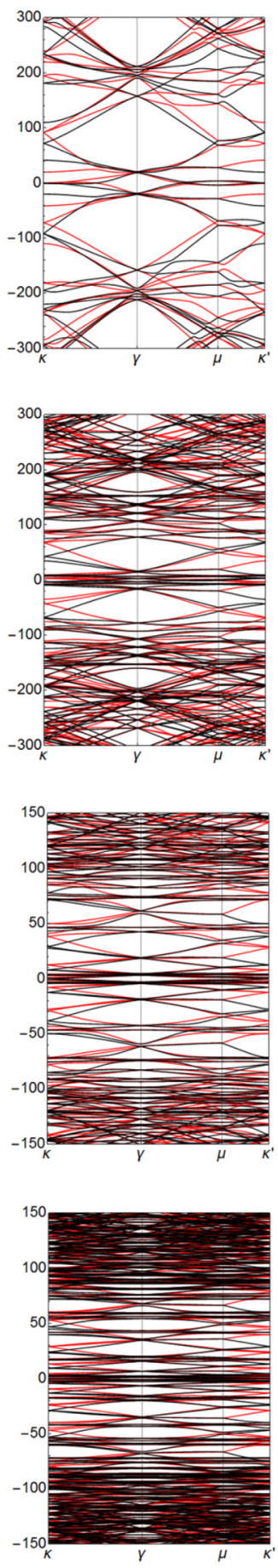

$\Delta=400 \mathrm{meV}$
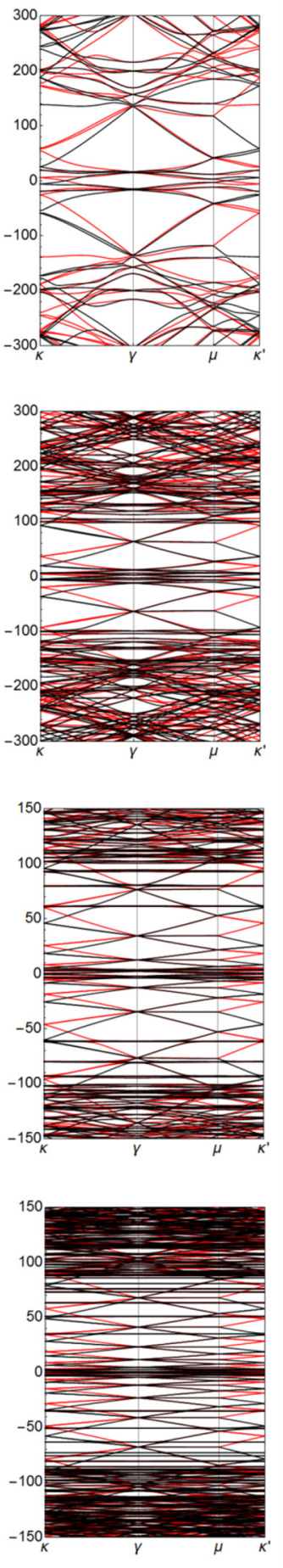

(b)

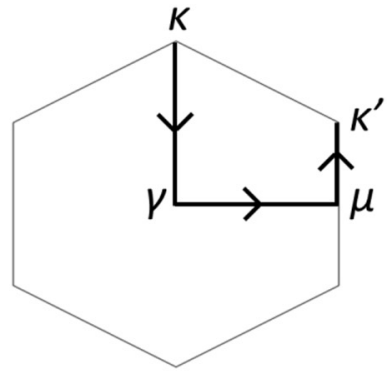

(c) $\theta=0.5^{\circ}, \Delta=400 \mathrm{meV}$

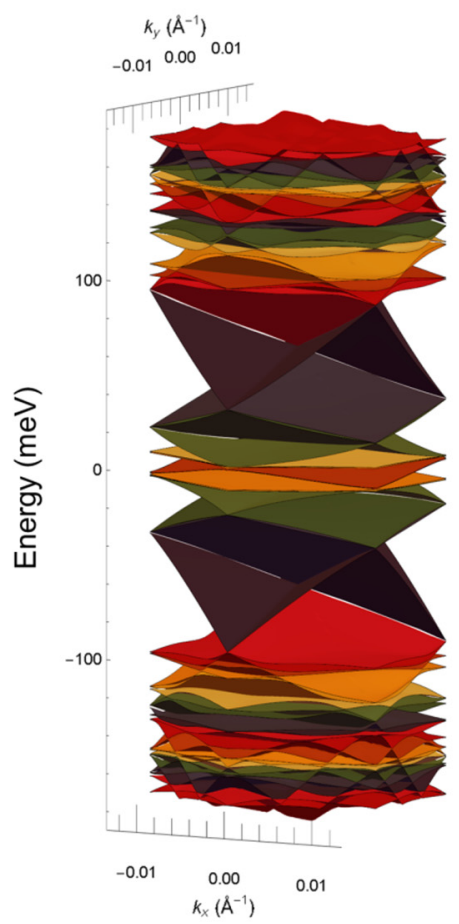

(d)

$$
\mathrm{E}_{\mathrm{F}}=50 \mathrm{meV}
$$

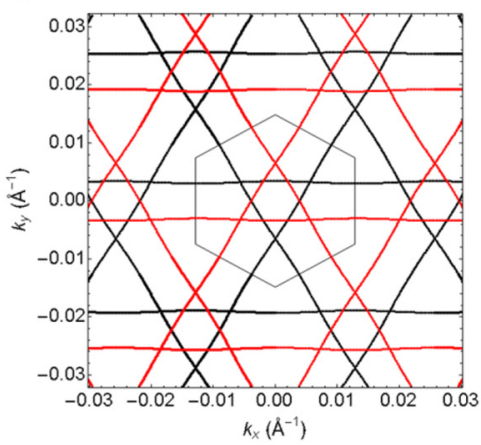

FIG. 2. (a) Band structure of the twisted bilayer at various twist angles and varying $\Delta$, calculated using the continuum model. (b) The moiré Brillouin zone. (c) A three-dimensional plot of $K$-valley bands and (d) the contour plot at $E_{F}=50 \mathrm{meV}$, calculated for $\theta=0.5^{\circ}$ and $\Delta=400 \mathrm{meV}$. The black and red lines represent the $K$ and $K^{\prime}$ valleys, respectively.

Hamiltonian is given by

$$
H=-\sum_{i, j} t\left(\mathbf{R}_{i}-\mathbf{R}_{j}\right)\left|\mathbf{R}_{i}\right\rangle\left\langle\mathbf{R}_{j}\right|+\text { H.c. },
$$

where $\mathbf{R}_{i}$ is the atomic coordinate, $\left|\mathbf{R}_{i}\right\rangle$ is the wave function at site $i$, and $t\left(\mathbf{R}_{i}-\mathbf{R}_{j}\right)$ is the transfer integral between atoms $i$ and $j$. We adopt the Slater-Koster-type formula for the transfer integral [55],

$$
-t(\mathbf{d})=V_{p p \pi}(d)\left[1-\left(\frac{\mathbf{d} \cdot \mathbf{e}_{z}}{d}\right)^{2}\right]+V_{p p \sigma}(d)\left(\frac{\mathbf{d} \cdot \mathbf{e}_{z}}{d}\right)^{2},
$$



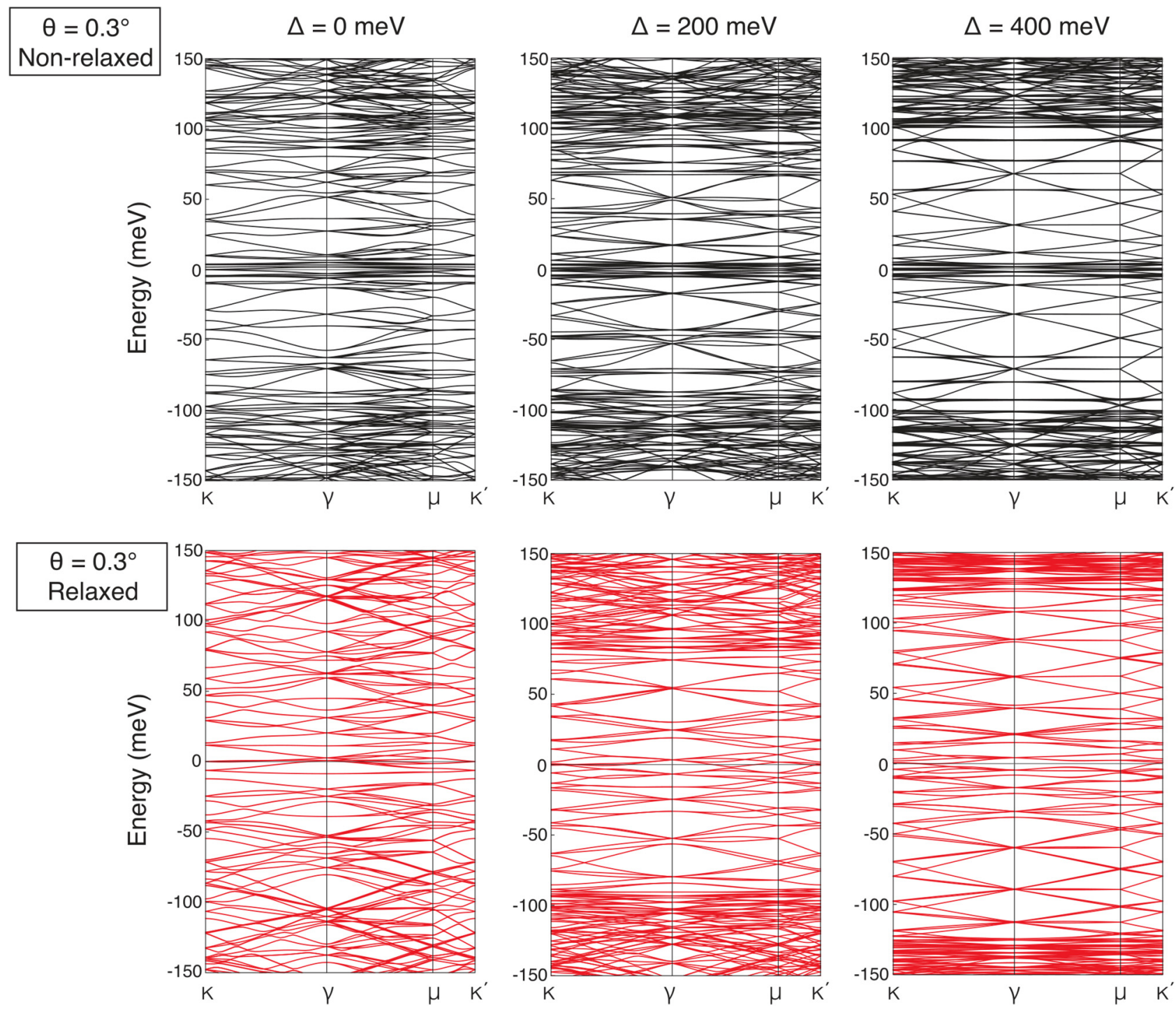

FIG. 3. Band structure of nonrelaxed (top panels) and relaxed (bottom panels) TBGs in $\theta=0.3^{\circ}$ and different $\Delta$ 's calculated using the tight-binding model.

$$
\begin{aligned}
& V_{p p \pi}(d)=V_{p p \pi}^{0} \exp \left(-\frac{d-a_{0}}{r_{0}}\right), \\
& V_{p p \sigma}(d)=V_{p p \sigma}^{0} \exp \left(-\frac{d-d_{0}}{r_{0}}\right),
\end{aligned}
$$

where $\mathbf{d}=\mathbf{R}_{i}-\mathbf{R}_{j}$ is the distance between two atoms and $\mathbf{e}_{z}$ is the unit vector on the $z$ axis. $V_{p p \pi}^{0} \approx-2.7 \mathrm{eV}$ is the transfer integral between nearest-neighbor atoms of monolayer graphene which are located at distance $a_{0}=a / \sqrt{3} \approx$ $0.142 \mathrm{~nm}, V_{p p \sigma}^{0} \approx 0.48 \mathrm{eV}$ is the transfer integral between two nearest vertically aligned atoms, and $d_{0} \approx 0.334 \mathrm{~nm}$ is the interlayer spacing. The decay length $r_{0}$ of the transfer integral is chosen to be $0.184 a$ so that the next-nearest intralayer coupling becomes $0.1 V_{p p \pi}^{0}$. At $d>\sqrt{3} a$, the transfer integral is very small and negligible. The optimized atomic positions are obtained by the method introduced in a previous work [50]. Using this, we construct the tight-binding Hamiltonian of the relaxed TBG and calculate the energy bands.
Figure 3 compares the electronic band structure of nonrelaxed (top panels) and relaxed (bottom panels) TBGs for $\theta=0.3^{\circ}$ and different $\Delta$ 's. In the tight-binding model, the valleys are not distinguished. We see that the energy bands of the nonrelaxed calculation quantitatively agree with those in the continuum method in Fig. 2. In the presence of the relaxation, we confirm that the qualitative feature remains the same: we still see the energy windows and the perfect 1D eigenmodes. The major difference from the nonrelaxed state is that the central pseudo-Landau levels mentioned in the previous section are completely hybridized with $1 \mathrm{D}$ eigenmodes and become a part of the dispersive bands. Also we notice that the bands in the zero-energy cluster become less flat and a bit more dispersive.

Figure 4 shows typical wave functions in the energy window in the nonrelaxed TBG and the relaxed TBG of $\theta=0.55^{\circ}$ and $\Delta=400 \mathrm{meV}$. Here we chose the corresponding states in nonrelaxed and relaxed cases, which are connected by a continuous increase of the relaxation. The state is chosen from a $1 \mathrm{D}$ band from the electron side with the velocity along the 


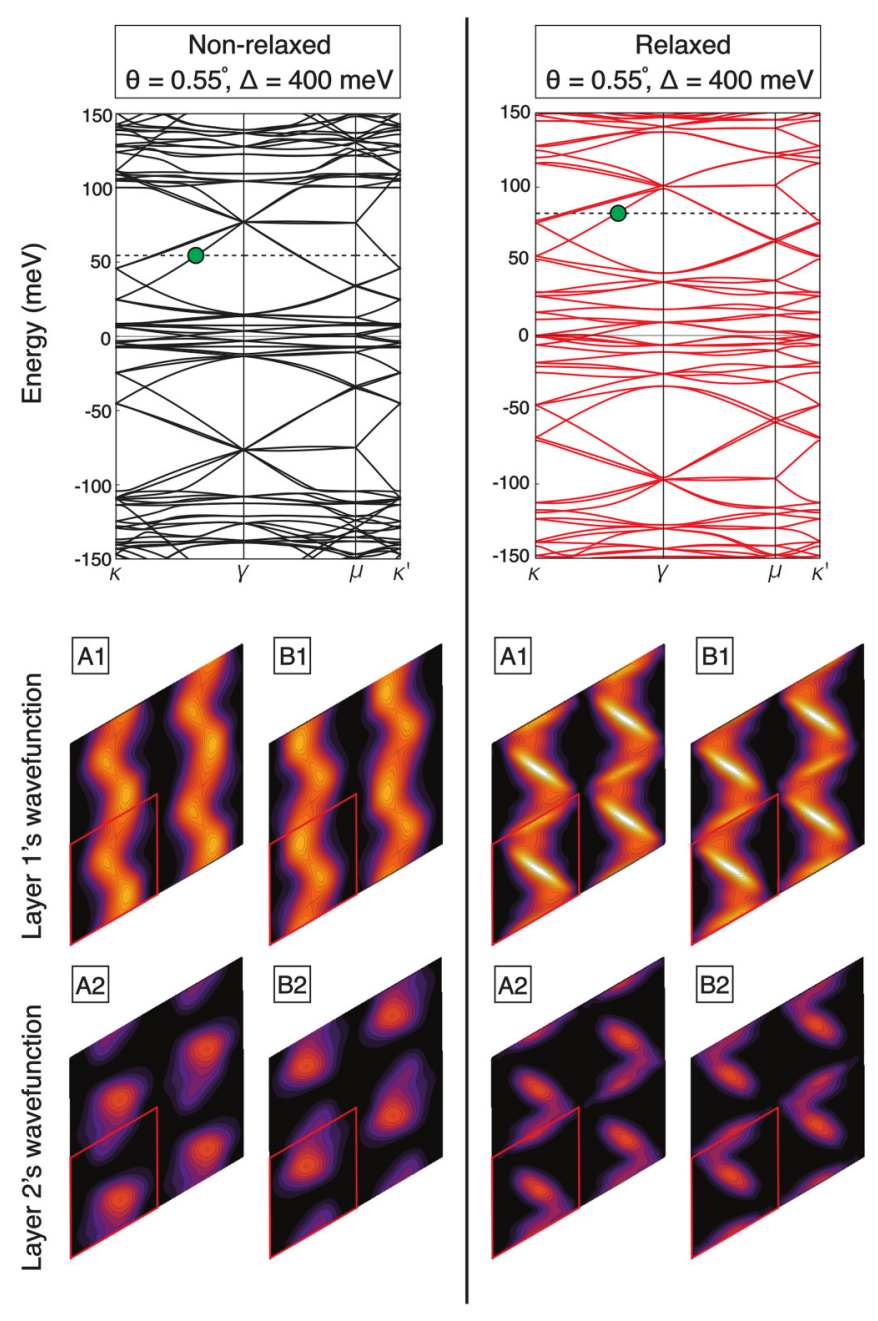

FIG. 4. Energy spectrum and the wave function of a typical state at the $K$ valley in the energy window (indicated in the spectrum) calculated for the nonrelaxed TBG and the relaxed TBG of $\theta=0.55^{\circ}$ and $\Delta=400 \mathrm{meV}$. The plot of the wave function represents the squared amplitude on sublattices $A_{1}, B_{1}, A_{2}$, and $B_{2}$ separately, where a red rhombus indicates a single moiré unit cell.

$k_{y}$ axis (the $\kappa-\gamma$ direction in Fig. 4). In each case, we observe that the wave function takes a 1D form extending along the $y$ direction, while it is disconnected in the perpendicular direction. The states in the different Fermi surface branches at the same energy are obtained by $\pm 120^{\circ}$ rotation of Fig. 4 . We confirm that the local current density is along the $-y$ direction, in accordance with the negative band velocity in the $k_{y}$ direction. The wave amplitude is mainly concentrated on layer 1 , while it is concentrated on layer 2 in the hole side states.

In the presence of the lattice relaxation, we see that the wave function becomes more localized on the AB-BA boundary. This is natural because the $\mathrm{AB}$ and $\mathrm{BA}$ regions (where the energy band is gapped out) significantly expand under the lattice relaxation, and the wave amplitude must be confined to the narrow boundary region [19,20]. A similar zigzag-shaped wave function was also reported in a recent work [19]. Interestingly, we see that the relaxed TBG's wave function in Fig. 4 has its amplitude not on the boundary along $y$ but only on the boundary in the two other directions along $(\sqrt{3} / 2,-1 / 2)$ and $(-\sqrt{3} / 2,-1 / 2)$. With closer inspection, we also find that it has different structures between the boundaries along $(\sqrt{3} / 2,-1 / 2)$ and $(-\sqrt{3} / 2,-1 / 2)$, although the atomic structures are completely symmetric. Recalling that each single boundary has two different traveling modes (denoted as modes 1 and 2) as mentioned, this result suggests that at every vertex of the triangular grid (AA region), mode 1 is always scattered to mode 2 in the $-120^{\circ}$ direction, while mode 2 is always scattered to mode 1 in the $+120^{\circ}$ direction. As a result, we have three independent zigzag traveling modes, as illustrated in Fig. 1(b).

\section{ORIGIN OF THE PERFECT 1D EIGENMODES}

The origin of the energy window and the $1 \mathrm{D}$ eigenmodes can be intuitively understood by a perturbational approach from the small interlayer coupling limit. Figure 5 shows the band structure of the continuum model in Eq. (1), with $\theta=0.3^{\circ}$ and $\Delta=400 \mathrm{meV}$ and with increasing interlayer coupling $u$ from zero to the actual value in TBG. With small $u$, we see that two gaps open on the electron side and the hole side, and they eventually become the window regions in the full $u$ parameter. We see that the $1 \mathrm{D}$ eigenmodes always remain inside the gap, preventing the spectrum from becoming fully gapped. The width of the energy window is obviously the order of $u$. The energy bands between the two gaps are squashed with increasing $u$ and finally become the zero-energy band cluster.

The opening of the two gaps for small $u$ can be explained by considering the following two-band model. For large $\Delta$, the low-energy region is dominated by the hole band of graphene layer 1 and the electron band of layer 2 . While considering the interlayer coupling $U$, we can imagine that the two opposite conical bands of single-layer graphenes are crossing with each other with a relative momentum shift of $\Delta \mathbf{K}_{j}(j=0,1,2)$, and the band anticrossing occurs at the cross section. Figure 6(a) illustrates the actual crossing lines between the Dirac cones in the case of $\theta=0.3^{\circ}$ and $\Delta=200 \mathrm{meV}$, where the three circles (red, blue, and green) correspond to $j=0,1,2$.

The size of the gap is roughly proportional to the matrix element of $U$ between the two states on the crossing line. Graphene's eigenstates are written in the $(A, B)$ spinor representation as

$$
|\mathbf{k}, s\rangle=\frac{1}{\sqrt{2}}\left(\begin{array}{c}
1 \\
-s e^{i \theta(\mathbf{k})}
\end{array}\right),
$$

where $s= \pm$ represent the conduction and valence bands, respectively, and $\theta(\mathbf{k})=\arctan \left(k_{y} / k_{x}\right)$. Now, the matrix element of $U$ from graphene 1 to graphene 2 is

$$
\left\langle\mathbf{k}+\Delta \mathbf{K}_{j},+|U| \mathbf{k},-\right\rangle \approx i u \sin \left[\theta(\mathbf{k})-\frac{2 \pi j}{3}\right],
$$

where $\left|\Delta \mathbf{K}_{j}\right| \ll|\mathbf{k}|$ is assumed. In Fig. 6(b), the thickness of the crossing lines represents the amplitude of the interlayer matrix element on the Dirac cones of layers 1 and 2, respectively. We see the matrix element vanishes near $E=0$, and this is the reason why the two major gaps open above and below $E=0$. For a small- $u$ limit, the number of states (per 


$$
\begin{aligned}
u= & 0.0103 \mathrm{eV} \\
& (10 \%)
\end{aligned}
$$

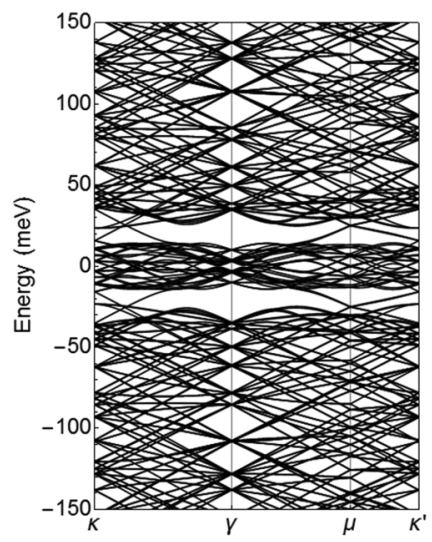

$0.0206 \mathrm{eV}$

$(20 \%)$

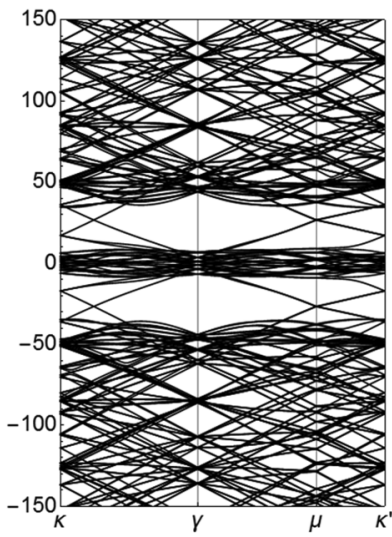

$0.0515 \mathrm{eV}$

$(50 \%)$

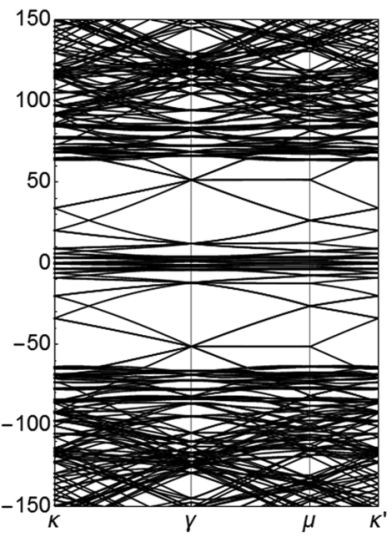

$0.103 \mathrm{eV}$

$(100 \%)$

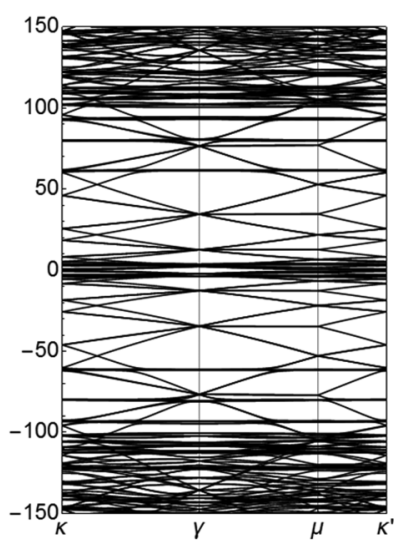

FIG. 5. Band structure of the continuum model for the TBG with $\theta=0.3^{\circ}$ and $\Delta=400 \mathrm{meV}$ and the interlayer coupling $u$ from zero to the actual value in TBG.

area) sandwiched by the two gaps is given by $2 n_{W}$, where

$$
n_{W}=g_{v} g_{s} \frac{\Delta}{4 \pi \hbar v} \Delta K,
$$

where $g_{v}=g_{s}=2$ are the spin and valley degeneracies and $\Delta K=\left|\Delta \mathbf{K}_{j}\right|=4 \pi \theta /(3 a)$. The parameter $n_{W}$ characterizes the typical carrier density to reach the energy window of the $1 \mathrm{D}$ eigenmodes. For $\theta=0.3^{\circ}$ and $\Delta=200 \mathrm{meV}$, for instance, we have $n_{W}=1.08 \times 10^{12} \mathrm{~cm}^{-2}$. Equation (12) is obtained by finding the density of states $D(E)=g_{v} g_{s} \Delta /\left(2 \pi \hbar^{2} v^{2}\right)$ for $|E|<\Delta / 2$ and multiplying by the maximum energy of the crossing line in Fig. 6(a), which is given by $\hbar v \Delta K / 2$. (a)

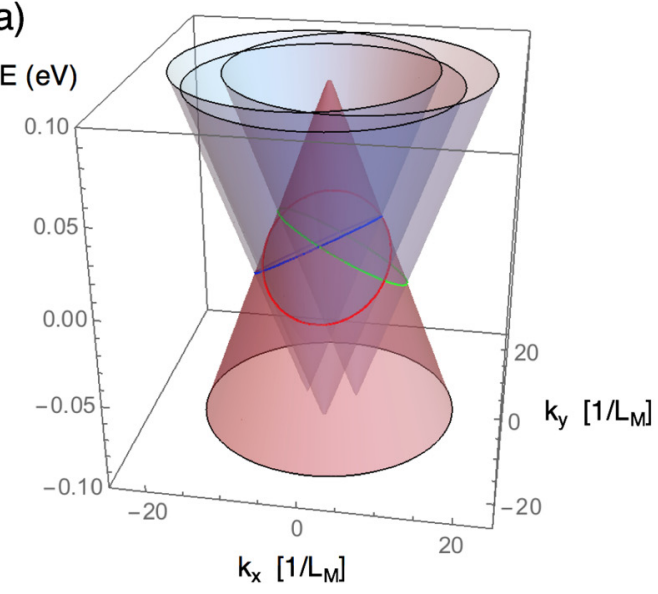

(b)
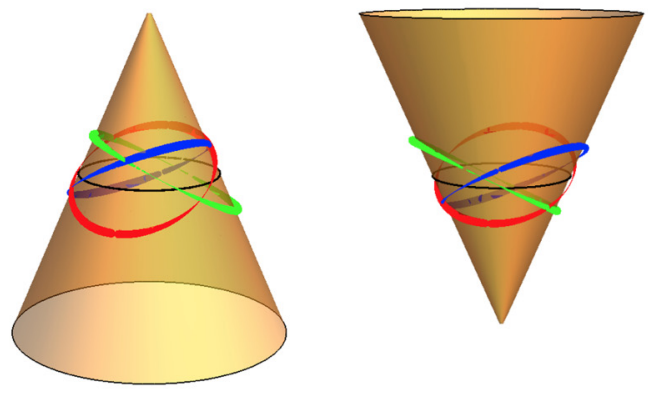
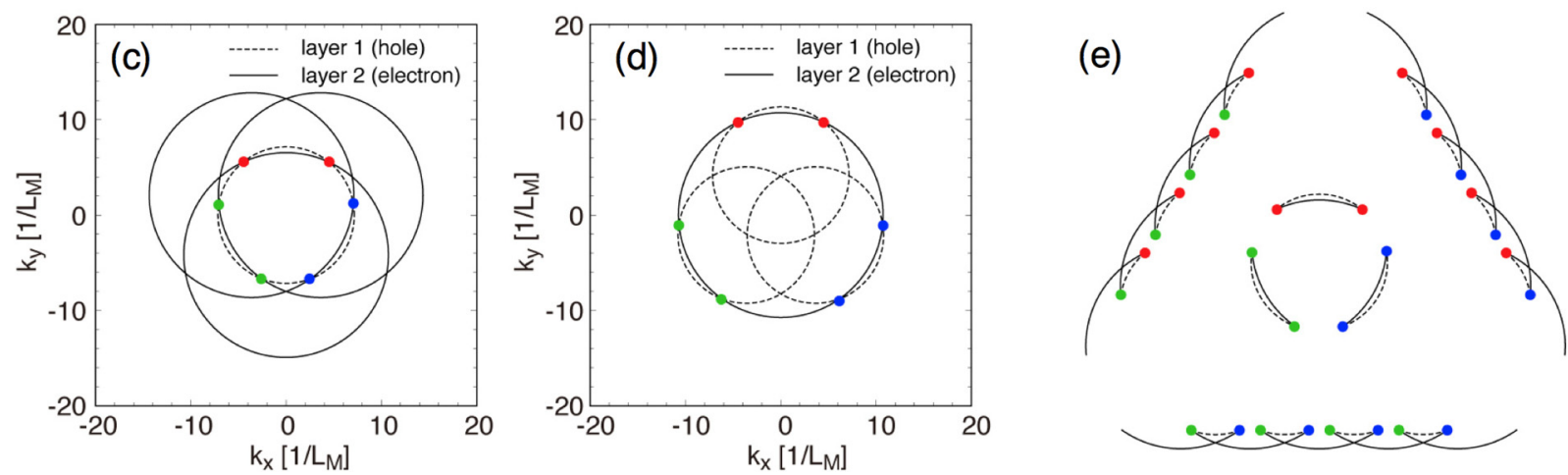

FIG. 6. (a) Crossing lines between the Dirac cones, where the three circles (red, blue, and green) correspond to $j=0,1,2$. Here we take $\theta=0.3^{\circ}$ and $\Delta=200 \mathrm{meV}$. (b) Amplitude of the interlayer matrix element on the crossing lines on the Dirac cones of layers 1 and 2. (c) and (d) Relative positions of the Fermi surfaces of layer 1 (dashed circles) and layer 2 (solid circles) in the absence of $u$ and at $E_{F}=20$ meV. (e) The hybridized Fermi surfaces after the infinitesimal anticrossing. 
The 1D eigenmodes remaining inside the energy window can be explained by the reconstruction of the Fermi surface. Figures 6(c) and 6(d) illustrate the Fermi surfaces before introducing $u$ at $E_{F}=20 \mathrm{meV}$, which is slightly below the maximum energy of the crossing rings. In Fig. 6(c), the central dashed circle represents the hole band of layer 1, and the three solid circles are the electron band of layer 2 with shifts $\Delta \mathbf{K}_{j}(j=0,1,2)$. In Fig. 6(d), the Fermi surface of layer 1 is centered instead. The hybridized Fermi surfaces after the infinitesimal anticrossing are shown in Fig. 6(e). We therefore have three open Fermi surfaces that are $120^{\circ}$ apart as well as three closed pockets. By increasing $u$, the closed pockets are gapped out due to a good nesting between the electron and hole parts. On the other hand, the open Fermi surfaces remain ungapped, which explains the origin of the 1D eigenmodes filling the gap. We also see that the open Fermi surface mainly consists of the layer 1 component (solid line), which is consistent with the fact that the wave function has a larger amplitude on layer 1 in Fig. 4.

In this picture, we consider only the band crossing of the first order in $u$, while we actually have high-order hybridization at other crossing points. It is somewhat surprising that the 1D eigenmodes in three directions are not hybridized and remain independent even for a large $u$ beyond the perturbational regime. This is understood by the $k$-space map of the interlayer matrix element in Fig. 7, where open circles represent graphene 1's hole states at $\mathbf{k}_{m_{1}, m_{2}}^{(1)}$, solid circles represent graphene 2's electron states at $\mathbf{k}_{m_{1}, m_{2}}^{(2)}$, and the bond thickness is proportional to the matrix element of $U$ between the two states. We can show that the 1D eigenmodes in the positive-energy window are contributed by graphene's states only in regions I, III, and V, and those in the negative-energy window are contributed by regions II, IV, and VI. This is consistent with the observation that the open Fermi surface in Fig. 6(e) consists of graphene's Fermi surface portions in the same regions. We see that the matrix element nearly vanishes on the boundary of different regions (dashed lines) according to Eq. (11), except for the $k$ points near the origin, which do not contribute to the low-energy states. As a result, the six regions I, II, ..., VI are nearly decoupled, and that is why the $1 \mathrm{D}$ eigenmodes running in the different directions remain independent with increasing $u$.

The vanishing matrix elements on the region boundaries can be explained by the pseudospin matching. For example, the single-layer eigenstates propagating in the $x$ direction are expressed by pseudospins $(1,1)$ and $(1,-1)$ for the hole band and the electron band, respectively. The interlayer matrix component of $j=0$ in Eq. (3) does not hybridize those two modes because $(1,1)$ and $(1,-1)$ are the eigenvectors of the matrix, and this is the reason why the vertical links (i.e., $j=0$ ) in Fig. 7 are almost vanishing on the $x$ axis, except for the vicinity of the origin. Similar arguments for $j=1,2$ explain the decoupling on the boundaries in the other two directions.

The perfect $1 \mathrm{D}$ eigenmodes in the biased TBG are analogous to those in zigzag graphene nanoribbons [56]. In a doped zigzag nanoribbon, it is known that each valley has different numbers of left-moving modes and right-moving modes at

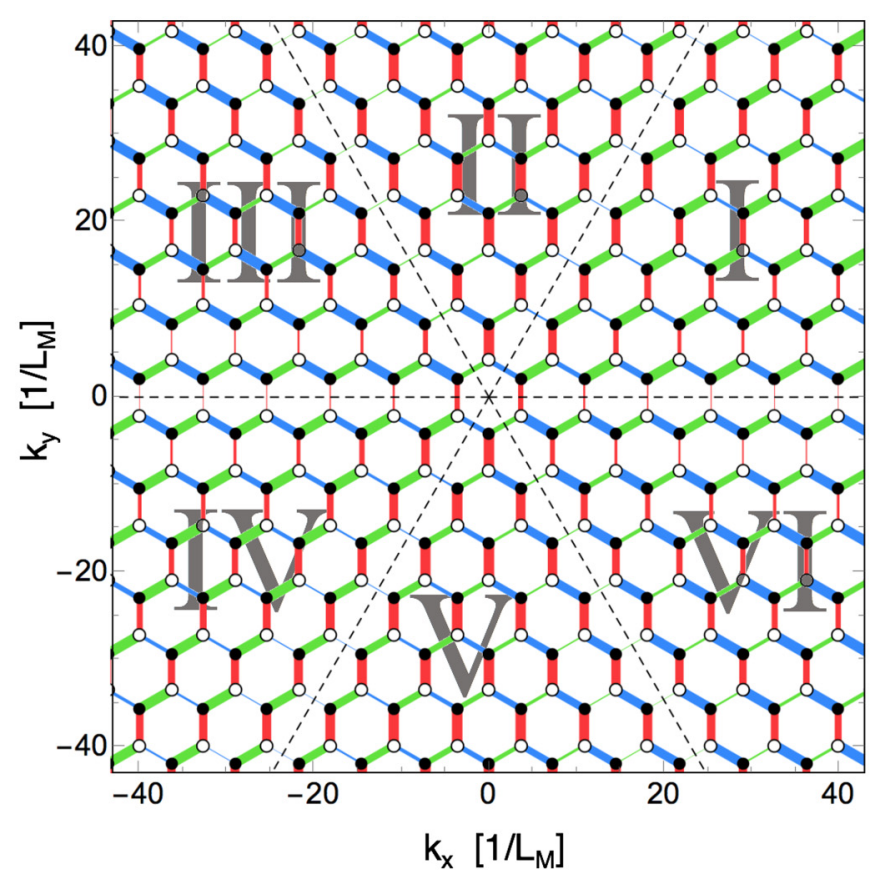

- layer 1 (hole)

layer 2 (electron)

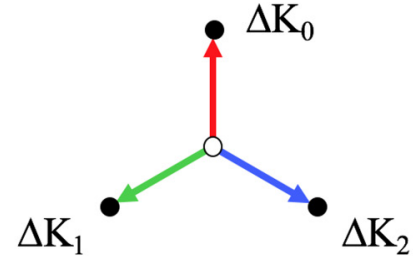

FIG. 7. Map of the interlayer matrix element, where open circles represent the hole states at $\mathbf{k}_{m_{1}, m_{2}}^{(1)}$ of single-layer graphene 1 and solid circles represent the electron states at $\mathbf{k}_{m_{1}, m_{2}}^{(2)}$ of graphene 2, where $\mathbf{k}=0$ is chosen in Eq. (5). The bond thickness is proportional to the matrix element of $U$ between the two states.

the Fermi energy: $n$ right modes and $n+1$ left modes in one valley and $n+1$ right modes and $n$ left modes in the other valley. The excess traveling mode in each valley remains a perfectly conducting channel even in the disordered system, as long as the impurities are long range and do not mix the different valleys. The perfect $1 \mathrm{D}$ eigenmodes in biased TBG can be viewed as a two-dimensional version of this, in that each single sector of I, III, and V has different numbers of outgoing modes and incoming modes (with respect to the $k$-space origin), as is clear from different arc lengths of the electron and hole Fermi surfaces in Fig. 6(e), and that different sectors are not hybridized by the interlayer coupling $u$. Therefore the excess modes originate from the electron Fermi surface, and they remain traveling modes even in the presence of $u$.

\section{DISCUSSION}

The disorder effect on these 1D eigenmodes is an important problem when considering the electronic transport. As shown in Fig. 1(b), each 1D mode is composed of straight parts on the AB-BA boundary and corner angles on AA spots. A hybridization of different $1 \mathrm{D}$ eigenmodes takes place only by a local mixing of modes 1 and 2 on the AB-BA boundaries 
or an irregular reflection at the AA corners. In real TBGs, the moiré structure exhibits a distorted triangular pattern with shifted AA spots and extended or shortened AB-BA boundaries [31-33], However, we expect that such a moiréscale distortion would not cause a strong mixing of different $1 \mathrm{D}$ eigenmodes because the local atomic structures of AA and AB-BA boundaries are not modified very much [57], such that the hubs and the links in the triangular lattice work in the same way as in the nondistorted system. Major scatterings should be mainly caused by short-range disorder smaller than the local structures of the AB-BA boundary and AA spots (which is about a few nanometers). A detailed study of the disorder scattering will be left for future works.

When the scattering can be neglected and the Fermi energy is in the energy window, the electronic transport must be dominated by the ballistic transport through the $1 \mathrm{D}$ eigenmodes. It is also expected that we do not have the Aharonov-Bohm (AB) oscillation in magnetic fields because the $1 \mathrm{D}$ eigenmodes do not enclose triangular domains and so do not cause any interferences. Recently, transport measurements were performed on small-angle TBGs under interlayer bias, and a significant $\mathrm{AB}$ oscillation was observed [34-36]. We expect that magnetic oscillations take place when the perfect $1 \mathrm{D}$ eigenmodes are not well formed, e.g., because the bias is not large enough or the Fermi energy is not in the corresponding region. To have perfect $1 \mathrm{D}$ eigenmodes, it is required that the energy window $\left(\left|E_{F}\right| \lesssim u\right)$ is dominated by the hole band of a single layer and the electron band of the other layer, and this gives the condition $\Delta / 2 \gtrsim u$, i.e., $\Delta \gtrsim 200 \mathrm{meV}$.

\section{CONCLUSION}

We used the continuum-model and tight-binding Hamiltonians to show that TBGs with an applied bias exhibit perfect $1 \mathrm{D}$ eigenmodes in well-defined energy windows on either side of zero energy. We found that these states never hybridize and they propagate independently in three different directions along the domain walls separating $\mathrm{AB}$ and $\mathrm{BA}$ regions. In the presence of arbitrary lattice deformations, we showed that the wave functions become even more localized on the domain boundaries. The formation of the well-defined energy windows and the origin of these states are explained by the twoband model consisting of the intersecting electron and hole bands of single-layer graphene, where the 1D eigenmodes correspond to the emergent open Fermi surfaces formed by the moiré interlayer hybridization.

\section{ACKNOWLEDGMENTS}

B.T. acknowledges financial support from the Japan Society for the Promotion of Science as a JSPS International Research Fellow (Summer Programme 2019), EPSRC Doctoral Training Centre Graphene NOWNANO EP/L01548X/1, and a Lloyd's Register Foundation Nanotechnology grant. M.K. acknowledges the financial support of JSPS KAKENHI Grant No. JP17K05496.
[1] J. M. B. Lopes dos Santos, N. M. R. Peres, and A. H. Castro Neto, Graphene Bilayer with a Twist: Electronic Structure, Phys. Rev. Lett. 99, 256802 (2007).

[2] E. J. Mele, Commensuration and interlayer coherence in twisted bilayer graphene, Phys. Rev. B 81, 161405(R) (2010).

[3] G. Trambly de Laissardière, D. Mayou, and L. Magaud, Localization of Dirac electrons in rotated graphene bilayers, Nano Lett. 10, 804 (2010).

[4] S. Shallcross, S. Sharma, E. Kandelaki, and O. A. Pankratov, Electronic structure of turbostratic graphene, Phys. Rev. B 81, 165105 (2010).

[5] E. Suárez Morell, J. D. Correa, P. Vargas, M. Pacheco, and Z. Barticevic, Flat bands in slightly twisted bilayer graphene: Tight-binding calculations, Phys. Rev. B 82, 121407(R) (2010).

[6] R. Bistritzer and A. H. MacDonald, Moiré bands in twisted double-layer graphene, Proc. Natl. Acad. Sci. USA 108, 12233 (2011).

[7] M. Kindermann and P. N. First, Local sublattice-symmetry breaking in rotationally faulted multilayer graphene, Phys. Rev. B 83, 045425 (2011).

[8] J. M. B. Lopes dos Santos, N. M. R. Peres, and A. H. Castro Neto, Continuum model of the twisted graphene bilayer, Phys. Rev. B 86, 155449 (2012).

[9] P. Moon and M. Koshino, Energy spectrum and quantum Hall effect in twisted bilayer graphene, Phys. Rev. B 85, 195458 (2012).
[10] G Trambly de Laissardière, D Mayou, and L Magaud, Numerical studies of confined states in rotated bilayers of graphene, Phys. Rev. B 86, 125413 (2012).

[11] Y. Cao, V. Fatemi, K. Fang, S. Watanabe, T. Taniguchi, E. Kaxiras, and P. Jarillo-Herrero, Unconventional superconductivity in magic-angle graphene superlattices, Nature (London) 556, 43 (2018).

[12] Y. Cao, V. Fatemi, A. Demir, S. Fang, S. L. Tomarken, J. Y. Luo, J. D. Sanchez-Yamagishi, K. Watanabe, T. Taniguchi, E. Kaxiras, R. C. Ashoori, and P. JarilloHerrero, Correlated insulator behavior at half-filling in magic-angle graphene superlattices, Nature (London) 556, 80 (2018).

[13] M. Yankowitz, S. Chen, H. Polshyn, Y. Zhang, K. Watanabe, T. Taniguchi, D. Graf, A. Young, and C. R. Dean, Tuning superconductivity in twisted bilayer graphene, Science $\mathbf{3 6 3}$, 1059 (2019).

[14] L. Xian, S. Barraza-Lopez, and M. Y. Chou, Effects of electrostatic fields and charge doping on the linear bands in twisted graphene bilayers, Phys. Rev. B 84, 075425 (2011).

[15] P. San-Jose and E. Prada, Helical networks in twisted bilayer graphene under interlayer bias, Phys. Rev. B 88, 121408(R) (2013).

[16] P. Moon, Y.-W. Son, and M. Koshino, Optical absorption of twisted bilayer graphene with interlayer potential asymmetry, Phys. Rev. B 90, 155427 (2014). 
[17] A. Ramires and J. L. Lado, Electrically Tunable Gauge Fields in Tiny-Angle Twisted Bilayer Graphene, Phys. Rev. Lett. 121, 146801 (2018).

[18] D. K. Efimkin and A. H. MacDonald, Helical network model for twisted bilayer graphene, Phys. Rev. B 98, 035404 (2018).

[19] M. Fleischmann, R. Gupta, F. Wullschläger, S. Theil, D. Weckbecker, V. Meded, S. Sharma, B. Meyer, and S. Shallcross, Perfect and controllable nesting in minimally twisted bilayer graphene, Nano Lett. 20, 971 (2020).

[20] N. R. Walet and F. Guinea, The emergence of one-dimensional channels in marginal-angle twisted bilayer graphene, 2D Mater. 7, 015023 (2019).

[21] T. Hou, Y. Ren, Y. Quan, J. Jung, W. Ren, and Z. Qiao, Current partition and conducting topological networks in twisted graphene bilayer, arXiv:1904.12826.

[22] E. McCann, Asymmetry gap in the electronic band structure of bilayer graphene, Phys. Rev. B 74, 161403(R) (2006).

[23] A. Vaezi, Y. Liang, D. H. Ngai, L. Yang, and E.-A. Kim, Topological Edge States at a Tilt Boundary in Gated Multilayer Graphene, Phys. Rev. X 3, 021018 (2013).

[24] F. Zhang, A. H. MacDonald, and E. J. Mele, Valley Chern numbers and boundary modes in gapped bilayer graphene, Proc. Natl. Acad. Sci. USA 110, 10546 (2013).

[25] M. Pelc, W. Jaskólski, A. Ayuela, and L. Chico, Topologically confined states at corrugations of gated bilayer graphene, Phys. Rev. B 92, 085433 (2015).

[26] L. Ju, Z. Shi, N. Nair, Y. Lv, C. Jin, J. Velasco, Jr., C. Ojeda-Aristizabal, H. A. Bechtel, M. C. Martin, A. Zettl et al., Topological valley transport at bilayer graphene domain walls, Nature (London) 520, 650 (2015).

[27] L.-J. Yin, H. Jiang, J.-B. Qiao, and L. He, Direct imaging of topological edge states at a bilayer graphene domain wall, Nat. Commun. 7, 11760 (2016).

[28] C. Lee, G. Kim, J. Jung, and H. Min, Zero-line modes at stacking faulted domain walls in multilayer graphene, Phys. Rev. B 94, 125438 (2016).

[29] J. Li, K. Wang, K. J. McFaul, Z. Zern, Y. Ren, K. Watanabe, T. Taniguchi, Z. Qiao, and J. Zhu, Gate-controlled topological conducting channels in bilayer graphene, Nat. Nanotechnol. 11, 1060 (2016).

[30] M. Koshino, Electron delocalization in bilayer graphene induced by an electric field, Phys. Rev. B 78, 155411 (2008).

[31] L. Brown, R. Hovden, P. Huang, M. Wojcik, D. A. Muller, and J. Park, Twinning and twisting of tri- and bilayer graphene, Nano Lett. 12, 1609 (2012).

[32] J. Lin, W. Fang, W. Zhou, A. R. Lupini, J. C. Idrobo, J. Kong, S. J. Pennycook, and S. T. Pantelides, AC/AB stacking boundaries in bilayer graphene, Nano Lett. 13, 3262 (2013).

[33] J. S. Alden, A. W. Tsen, P. Y. Huang, R. Hovden, L. Brown, J. Park, D. A. Muller, and P. L. McEuen, Strain solitons and topological defects in bilayer graphene, Proc. Natl. Acad. Sci. USA 110, 11256 (2013).

[34] P. Rickhaus, J. Wallbank, S. Slizovskiy, R. Pisoni, H. Overweg, Y. Lee, M. Eich, M.-H. Liu, K. Watanabe, T. Taniguchi, T. Ihn, and K. Ensslin, Transport through a network of topological channels in twisted bilayer graphene, Nano Lett. 18, 6725 (2018).

[35] H. Yoo, R. Engelke, S. Carr, S. Fang, K. Zhang, P. Cazeaux, S. H. Sung, R. Hovden, A. W. Tsen, T. Taniguchi, K. Watanabe, G.-C. Yi, M. Kim, M. Luskin, E. B. Tadmor, E. Kaxiras, and
P. Kim, Atomic and electronic reconstruction at the van der Waals interface in twisted bilayer graphene, Nat. Mater. 18, 448 (2019).

[36] S. G. Xu, A. I. Berdyugin, P. Kumaravadivel, F. Guinea, R. K. Kumar, D. A. Bandurin, S. V. Morozov, W. Kuang, B. Tsim, S. Liu, J. H. Edgar, I. V. Grigorieva, V. I. Fal'ko, M. Kim, and A. K. Geim, Giant oscillations in a triangular network of one-dimensional states in marginally twisted graphene, Nat. Commun. 10, 4008 (2019).

[37] S. Huang, K. Kim, D. K. Efimkin, T. Lovorn, T. Taniguchi, K. Watanabe, A. H. MacDonald, E. Tutuc, and B. J. LeRoy, Topologically Protected Helical States in Minimally Twisted Bilayer Graphene, Phys. Rev. Lett. 121, 037702 (2018).

[38] J. T. Chalker and P. D. Coddington, Percolation, quantum tunneling and the integer Hall effect, J. Phys. C 21, 2665 (1988).

[39] P. Moon and M. Koshino, Optical absorption in twisted bilayer graphene, Phys. Rev. B 87, 205404 (2013).

[40] M. Koshino, Interlayer interaction in general incommensurate atomic layers, New J. Phys. 17, 015014 (2015).

[41] M. Koshino and P. Moon, Electronic properties of incommensurate atomic layers, J. Phys. Soc. Jpn. 84, 121001 (2015).

[42] G. Tarnopolsky, A. J. Kruchkov, and A. Vishwanath, Origin of Magic Angles in Twisted Bilayer Graphene, Phys. Rev. Lett. 122, 106405 (2019).

[43] J. Liu, J. Liu, and X. Dai, Pseudo Landau level representation of twisted bilayer graphene: Band topology and implications on the correlated insulating phase, Phys. Rev. B 99, 155415 (2019).

[44] M. Koshino, N. F. Q. Yuan, T. Koretsune, M. Ochi, K. Kuroki, and L. Fu, Maximally Localized Wannier Orbitals and the Extended Hubbard Model for Twisted Bilayer Graphene, Phys. Rev. X 8, 031087 (2018).

[45] A. M. Popov, I. V. Lebedeva, A. A. Knizhnik, Y. E. Lozovik, and B. V. Potapkin, Commensurate-incommensurate phase transition in bilayer graphene, Phys. Rev. B 84, 045404 (2011).

[46] K. Uchida, S. Furuya, J.-I. Iwata, and A. Oshiyama, Atomic corrugation and electron localization due to moiré patterns in twisted bilayer graphenes, Phys. Rev. B 90, 155451 (2014).

[47] M. M. van Wijk, A. Schuring, M. I. Katsnelson, and A. Fasolino, Relaxation of moiré patterns for slightly misaligned identical lattices: Graphene on graphite, 2D Mater. 2, 034010 (2015).

[48] S. Dai, Y. Xiang, and D. J. Srolovitz, Twisted bilayer graphene: Moiré with a twist, Nano Lett. 16, 5923 (2016).

[49] S. K. Jain, V. Juričić, and G. T. Barkema, Structure of twisted and buckled bilayer graphene, 2D Mater. 4, 015018 (2016).

[50] N. N. T. Nam and M. Koshino, Lattice relaxation and energy band modulation in twisted bilayer graphene, Phys. Rev. B 96, 075311 (2017).

[51] S. Carr, D. Massatt, S. B. Torrisi, P. Cazeaux, M. Luskin, and E. Kaxiras, Relaxation and domain formation in incommensurate two-dimensional heterostructures, Phys. Rev. B 98, 224102 (2018).

[52] X. Lin, D. Liu, and D. Tománek, Shear instability in twisted bilayer graphene, Phys. Rev. B 98, 195432 (2018).

[53] F. Guinea and N. R. Walet, Continuum models for twisted bilayer graphene: Effect of lattice deformation and hopping parameters, Phys. Rev. B 99, 205134 (2019).

[54] P. Lucignano, D. Alfè, V. Cataudella, D. Ninno, and G. Cantele, Crucial role of atomic corrugation on the flat bands and energy 
gaps of twisted bilayer graphene at the magic angle $\theta \sim 1.08^{\circ}$, Phys. Rev. B 99, 195419 (2019).

[55] J. C. Slater and G. F. Koster, Simplified LCAO method for the periodic potential problem, Phys. Rev. 94, 1498 (1954).
[56] K. Wakabayashi, Y. Takane, and M. Sigrist, Perfectly Conducting Channel and Universality Crossover in Disordered Graphene Nanoribbons, Phys. Rev. Lett. 99, 036601 (2007).

[57] M. Koshino and Y.-W. Son, Moiré phonons in twisted bilayer graphene, Phys. Rev. B 100, 075416 (2019). 